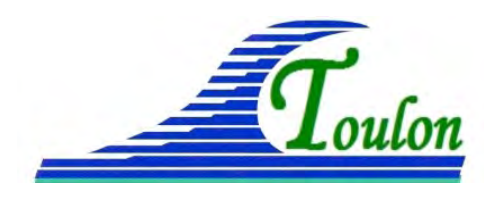
XIV èmes Journées Nationales Génie Côtier - Génie Civil Toulon, 29 juin au $1^{\text {er }}$ juillet 2016

DOI:10.5150/jngcgc.2016.018 @ Editions Paralia CFL disponible en ligne - http://www.paralia.fr - available online

\title{
Caractérisation de la dynamique sédimentaire le long des sites littoraux du bassin versant côtier de la région de Ksar Esghir (Tanger, Maroc)
}

\author{
Hanaa BENALI ${ }^{1}$, Brahim EL MOUTCHOU ${ }^{1}$
}

1. Equipe de Recherche en Géologie et Océanologie (ERGO/UAE/E02FS), Département de Géologie, Faculté des Sciences. BP 2121, M’hannech. 2. 93000 Tétouan, Maroc.hanabena@gmail.com ; brahimelmoutchou@yahoo.fr

\section{Résumé :}

La caractérisation de la dynamique sédimentaire de la frange littorale couvrant les différents sites du bassin versant côtier de la région de Ksar Esghir, comprise entre les plages Lmarssa à l'Est et Playa Blanca à l'Ouest, est entreprise dans le but de qualifier le contexte morphodynamique et morphosédimentaire à travers l'analyse granulométrique et l'application du test de Visher pour les modes de transport. Ainsi, cette étude a permis de mettre en évidence, les faits suivants :

- Une dominance des sables moyens le long des différents sites couvrant du bassin versant côtier de la région de Ksar Esghir;

- Un brassage mécanique, des sédiments littoraux, suite à la superposition dans le temps des effets des dynamiques marine et fluviale;

- Une évolution à composante (E-W), avec une répartition des sables selon un gradient décroissant de l'Est (Sables moyens) à l’Ouest (Sables fins), régi par les courants de houles provenant du secteur Est;

- Un transport et un triage sous l'effet du courant de dérive littorale, avec un gradient décroissant des sables de l'Est vers l'Ouest,

- Une dominance du mode transport par saltation associé à la suspension.

Mots-clés : Littoral, Dynamique sédimentaire, Test de Visher, Ksar Esghir, Tanger, Maroc.

\section{Introduction}

Les écosystèmes côtiers sont des interfaces en perpétuel changement dans le temps et dans l'espace. Ils sont soumis à des influences continentales et marines. De ce fait, ils sont des exutoires naturels des bassins versants continentaux et reçoivent par lessivage des sols, des eaux enrichies des divers produits de l'érosion.

Ainsi et en fonction de la dynamique régnante dans chaque portion du littoral, la tendance générale, est souvent une tendance de régularisation par redistribution latérale des matériaux. Dans les situations stables, la redistribution se réalise selon un bilan conservant à chaque secteur un "budget" sédimentaire et toute modification de ces 
échanges aboutit à des perturbations dans l'équilibre local et à l'apparition des phénomènes d'instabilité.

Dans ce sens, le littoral marocain de Ksar Esghir, offre des potentialités d'investigation plus ou moins importante selon le tracé et la nature lithologique du rivage et le relief continentale frangeant, d'où l'obligation de la réalisation d'une analyse granulométrique détaillée des sédiments superficiels des plages du littoral en question.

\section{Caractères généraux de la zone d'étude}

Le littoral du bassin versant côtier de la région de Ksar Esghir, de direction générale $\mathrm{E}$ W, se situé au NW du Maroc sur le détroit du Gibraltar entre Tanger et Sebta (figure 1). Elle est limitée à l'Est par la ville de Sebta, à l'Ouest par la ville de Tanger, au Nord par le Détroit de Gibraltar et au Sud par les formations géologiques du Rif externe.

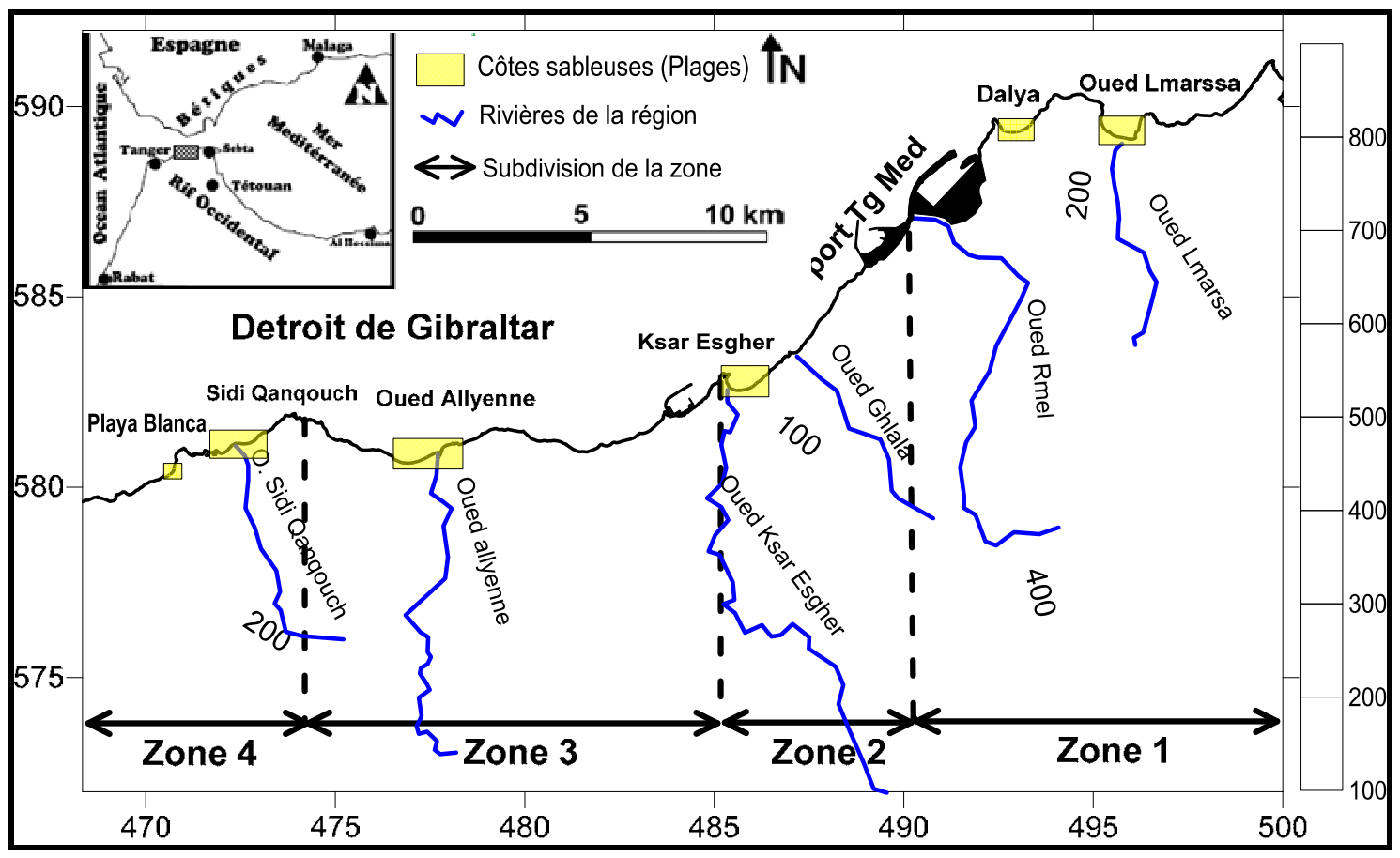

Figure 1. Carte de localisation des plages sableuses sur la frange littorale étudiée.

La zone d'étude occupe l'extrémité Nord de la chaine rifaine, qui est l'un des segments de la chaîne alpine constituant le tronçon Sud de l'arc de Gibraltar (DURAND DELGA et al., 1960-1962 ; DIDON et al., 1973 ; DURAND DELGA \& FONTEBOTE, 1980). Elle constitue une entité géologique à trois domaines distincts : Le domaine interne, le domaine des nappes des flyschs et le domaine externe (CHALOUAN, 1986 ; PAIDAR.MED, 1996).

L'aspect morphologique des éléments de reliefs de la région considérée, permet la distinction du caractère montagneux à trois composantes géomorphologiques, à savoir : 


\section{XIV èmes Journées Nationales Génie Côtier - Génie Civil \\ Toulon, 29 juin au $1^{\text {er }}$ juillet 2016}

Les zones hautes, les zones de plaines et les zones côtières (BENALI \& EL MOUTCHOU, 2013).

Le climat de type méditerranéen tempéré et chaud permet la distinction de deux saisons distinctes : Un hiver frais et humide aux précipitations violentes entre Octobre et Avril et un été chaud et sec de Mai à Septembre. Ce contexte climatique est caractérisé par une pluviométrie moyenne annuelle d'environ $800 \mathrm{~mm}$, une température moyenne annuelle de $18^{\circ} \mathrm{C}$ et des vents à deux composantes Est et Ouest (figure 2).

Le contexte météo-marin est marqué par des houles en provenance de deux secteurs (JAAIDI et al., 1993 ; EL MOUTCHOU, 2014) (figures 2 et 3) avec :

- des houles Ouest à NW, dominantes d'origine atlantique sur la partie occidentale, de période varient entre 9 et 12 sec et d'amplitude moyenne de $2 \mathrm{~m}$.

- des houles des houles du secteur Est à NE, dues à des mers de vent locales, caractérisées par des périodes courtes, de 3 à 5 sec et des hauteurs inférieures à 1,5 m. Ces houles attaquent les littoraux à l'Est avec une incidence de $20^{\circ}$ à $30^{\circ}$, engendrant ainsi, un transfert latéral vers l'Ouest.

La marée, généralement très faible, provient de l'Atlantique et se propage vers la Méditerranée à travers le détroit de Gibraltar. Elle est de type semi-diurne à faible marnage qui décroît de l'Ouest vers l'Est (EL MOUTCHOU, 1995 \& 2014; EL MOUTCHOU et al., 2011a \& 2011b).
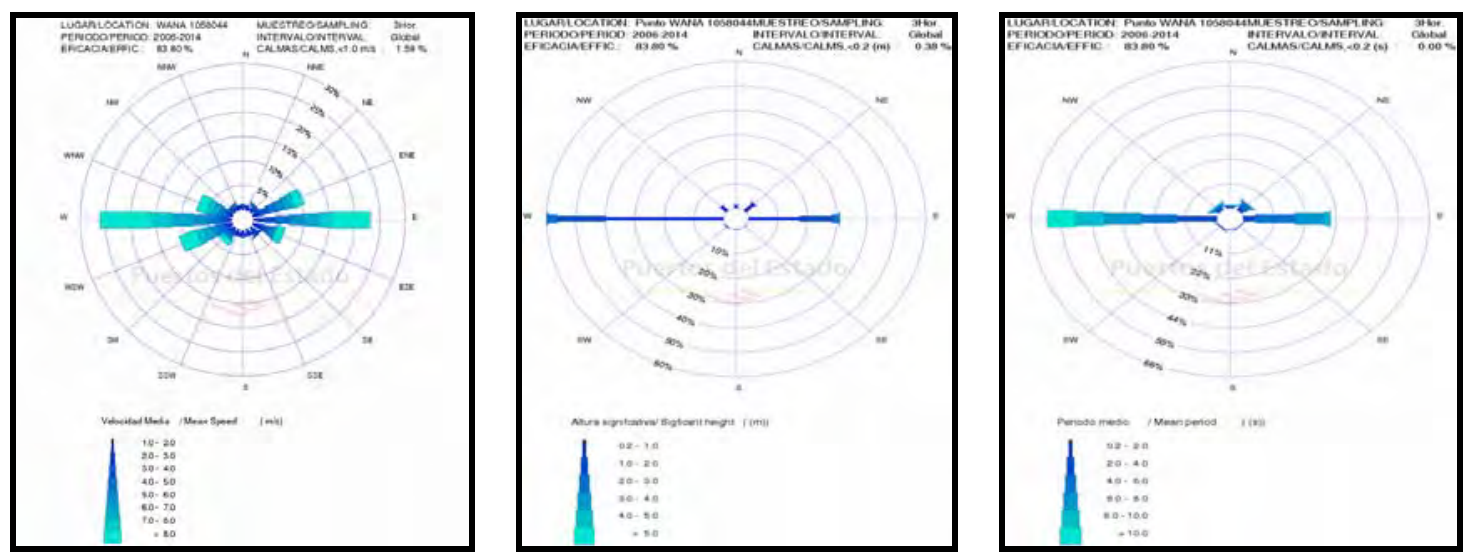

Figure 2. Rose des vents et Direction des houles, Hauteurs significatives et périodes correspondantes au Point1058044 entre 2006 et 2014 (http://www.puertos.es/es/oceanografia_y_meteorologia). 

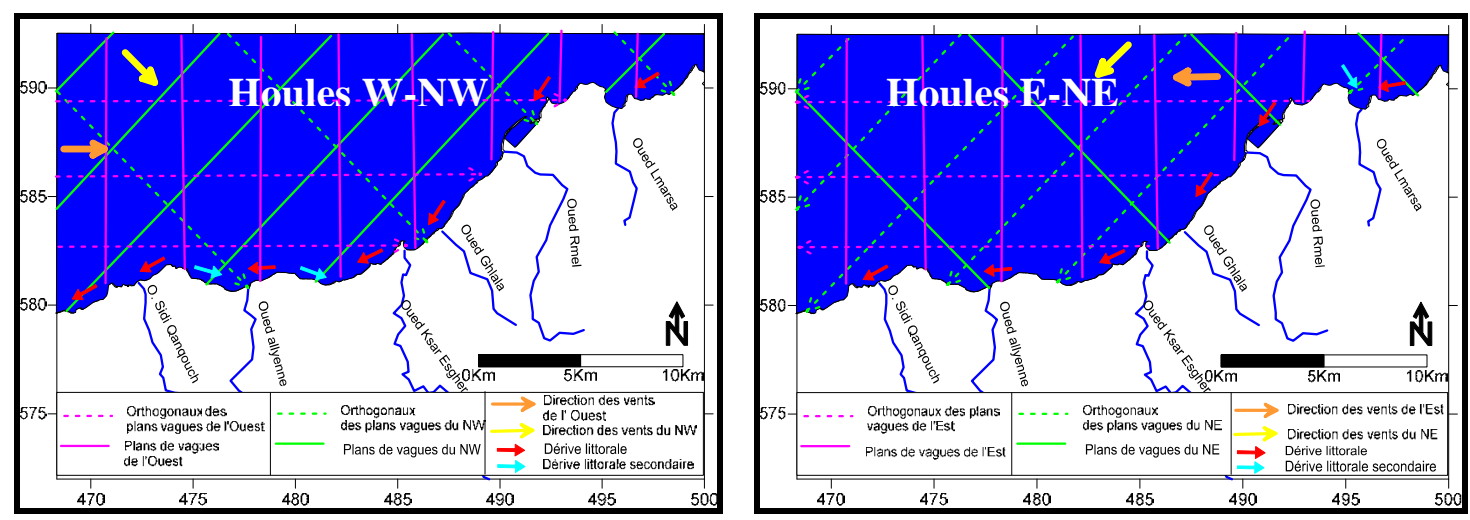

Figure 3. Direction des houles et des courants latéraux dans la zone côtière de Ksar

Esghir.

\section{Matériel et méthodes}

La caractérisation sédimentologique des sédiments superficiels du littoral de Ksar Esghir a été effectuée par l'analyse des échantillons des plages en fonction du changement de faciès granulométrique (EL FADEL, 2006 ; EL MOUTCHOU, 1995 \& 2014 ; EL MOUTCHOU et al., 2011a). Le traitement des échantillons sableux (98 échantillons, prélevés selon 31 radiales perpendiculaires au trait de côte actuel), par tamisage à sec sur une série de 9 tamis AFNOR allant de $2.5 \mathrm{~mm}$ à $0.063 \mathrm{~mm}$, a permis la mise en évidence des résultats ci-dessous et le calcul des paramètres de distribution selon FOLK et WARD (1957).

Les modes de transport ont été déterminés selon le Test de VISHER (1969), qui se base sur l'interprétation des données granulométriques. Cette méthode permet de définir trois modes de transport, à savoir : Le charriage/Roulement, la saltation et la suspension.

Ainsi, l'ensemble des résultats, des représentations et des calculs associés, seront regroupée selon quatre zones, de l'Est vers l'Ouest, à savoir : Zone. 1 (Plages d'Oued Lmarssa et Dalya), Zone. 2 (Plage Ksar Esghir), Zone. 3 (Plage Oued Allyenne) et Zone. 4 (Plages de Sidi Qanqouch et Playa Blanca).

\section{Résultats et discussions}

4.1 Répartition superficielles des sables des plages de la région de Ksar Esghir

La détermination des moyennes granulométrique (MZ) des sables le long de la frange littorale du bassin versant côtier de Ksar Esghir comprise entre les plages Oued Lmarssa et Playa Blanca, montre un éventail granulométrique, le long des diverses zones considérées, allant des sables fins aux grossiers, avec une dominance nette des sables moyens, avec (figure 4) :

- Une dominance nette des sables moyens à fins de diamètre allant de 1 et 2,4 $\Phi$, occupant la superficie de toutes les zones (1, 2, 3 et 4); 


\section{XIV ${ }^{\text {èmes }}$ Journées Nationales Génie Côtier - Génie Civil \\ Toulon, 29 juin au $1^{\text {er }}$ juillet 2016}

- Un gradient granulométrique en allant de l'Est (Sables moyens) vers l'Ouest (Sables fins), surtout dans les zones (2 et 3). Ce qui est dû aux effets de la dérive littorale $\mathrm{E}$ - W;

- Un hydrodynamisme reflétant des conditions d'agitation plus ou moins constantes.

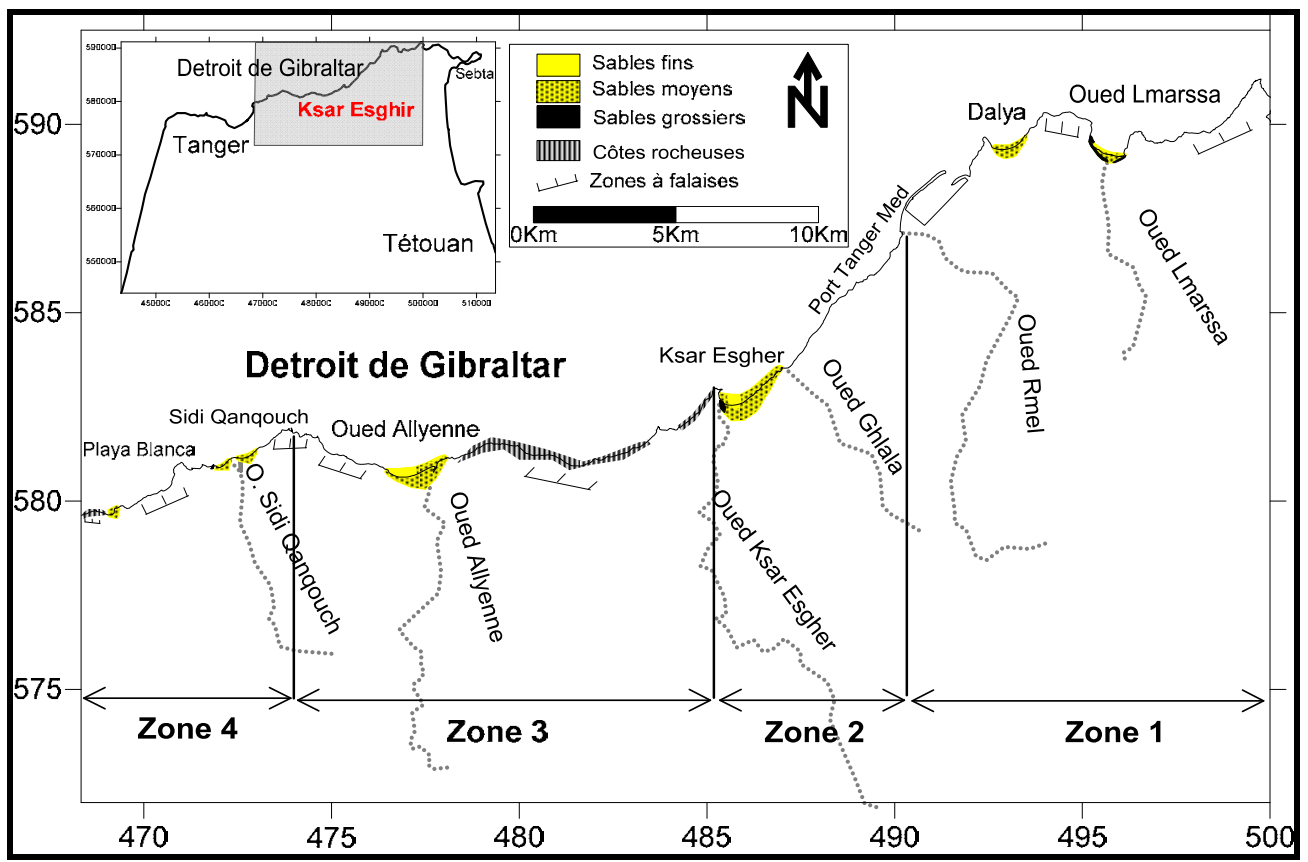

Figure 4. Synthèse de la répartition superficielle des matériaux sur le littoral de Ksar Esghir.

4.2 Modes de transport des sédiments superficiels le long des plages de Ksar Esghir

L'application du test de Vischer sur les matériaux des plages sableuses de la frange littorale du bassin versant côtier de Ksar Esghir entre Oued Lamrssa et Lamnar, montre selon le zonage adopté, de l'Est vers l'Ouest, les faits suivants :

- Zone. 1, montre des sables mal classée au niveau de la plage Oued Lmarssa ou prédomine la fraction moyenne avec un mode de transport dominant par saltation d'environ 90\% des particules, associé à des populations transportées par suspension. Tandis qu'au niveau de la plage Dalya (Zone. 1), les sables à dominance moyenne, montre un classement bon à modéré où les matériaux sont transportés par saltation (Plus de 60\%) et par suspension (Moins de 40\%, figure 5). Cette répartition des sables traduit dans l'ensemble, des conditions hydrodynamiques de moyenne énergie.

- Zones (2, 3 et 4), le long des plages de Ksar Esghir, Oued Allyenne, Sidi Qanqouch et Playa Blanca (figure 6), avec, des sables bien à modérément classées où les sédiments moyens à fins prédominent. Le test de Vischer dans ces zones, montre que le transport des particules se fait essentiellement par saltation (Pour plus de 50\% des particules) et suspension (pour moins de $50 \%$ des particules). Ceci indique également des conditions hydrodynamiques moyennes dans l'ensemble. 

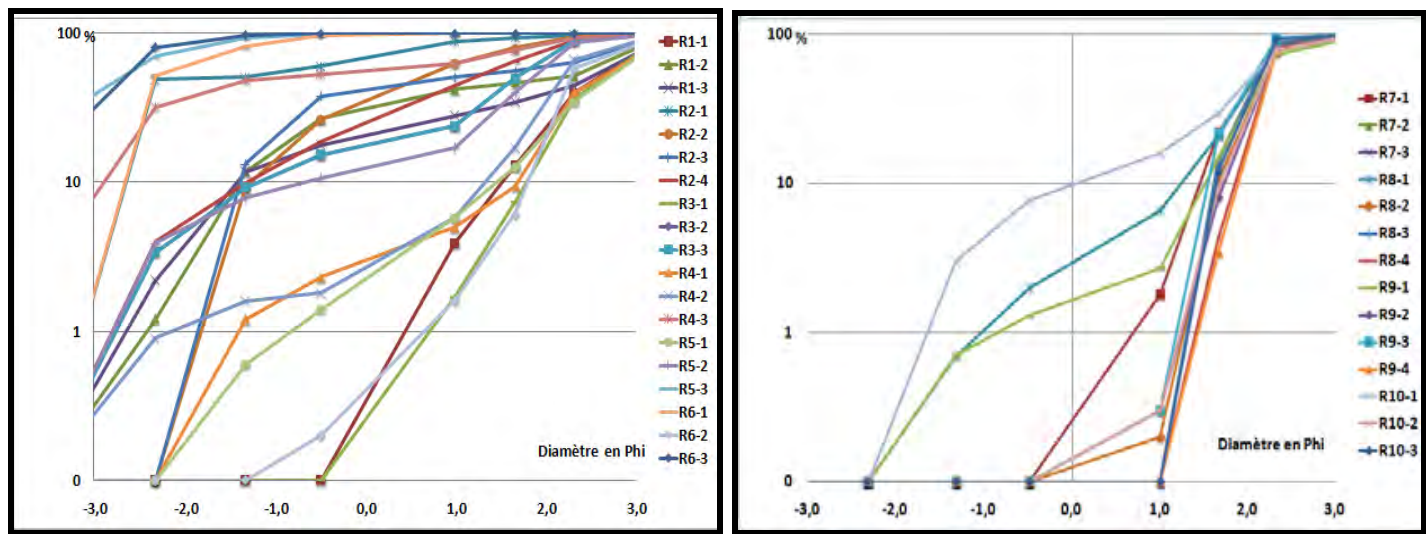

Figure 5. Test de Vischer des matériaux des plages d'Oued Lmarssa et Dalya.
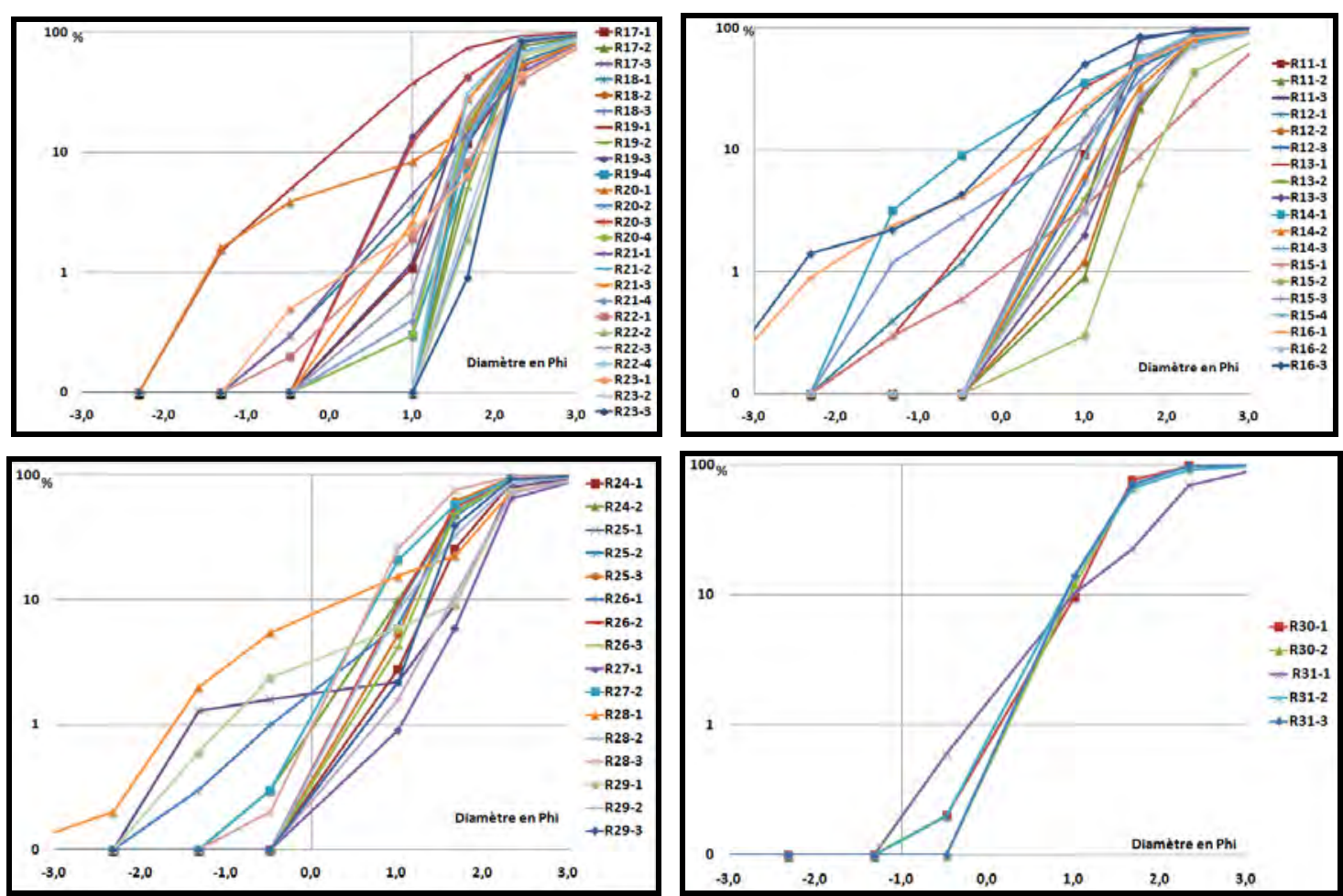

Figure 6. Test de Vischer des matériaux des plages Ksar Esghir, Oued Allyenne, Sidi Qanqouch et Playa Blanca.

\section{Conclusions}

La caractérisation sédimentologique des 98 échantillons des sédiments superficiels prélevés sur les plages du bassin versant côtier de la région de Ksar Esghir, selon 31 radiales perpendiculaires à la ligne de rivage et couvrant l'ensemble des sites sableux d’Oued Lmarssa, Dalya, Ksar Esghir, Oued Allyenne, Sidi Qanqouch et Playa Blanca, a permis la qualification des conditions hydrodynamiques contrôlant la répartition spatiale, le transport et le sens de déplacement des stocks sédimentaires dans chacune des zones considérées. 


\section{XIV èmes Journées Nationales Génie Côtier - Génie Civil \\ Toulon, 29 juin au $1^{\text {er }}$ juillet 2016}

Ainsi, les stocks sédimentaires des six plages étudiées (Zones. 1 - 2 - 3 et 4), présente une répartition sédimentaire assez homogène constituée essentiellement par des sables moyens, modérément à bien classés, transportés essentiellement par saltation.

Cette situation caractérise les sites à pente moyenne, soumises à un hydrodynamisme moyen à composante temporelle constante agitation (EL MOUTCHOU, 1995 \& 2014 ; MASSELINK \& HEGGE, 1995 ; NORDSTROM, 1977), tel est le cas des différents sites de la zone étudiée. De même, l'existence de sédiments grossiers sous forme de bandes localisées transportées par charriage correspond en général, à de brusque décharge détritique indiquant diminution de l'énergie du courant transporteur (EL MOUTCHOU, 1995).

En conclusion, le contexte morphodynamique de la frange littorale du bassin versant côtier de Ksar Esghir, montre :

- Un brassage mécanique, des sédiments littoraux, suite à la superposition dans le temps des effets des dynamiques marine et fluviale;

- Une évolution à composante (E-W), avec une répartition des sables selon un gradient décroissant de l'Est (Sables moyens) à l'Ouest (Sables fins), régi par les courants de houles provenant du secteur Est;

- Un transport et un triage sous l'effet du courant de dérive littorale, avec un gradient décroissant des sables de l'Est vers l'Ouest.

Par comparaison avec la zone côtière de la ville de Tétouan (Plages de Fnideq, Restinga Smir, Kabyla, M’diq, Cabo Negro, Martil, Sidi Abdeslam, Azla et Emsa), à l'Est de la région de Ksar Esghir (EL MOUTCHOU, 2014), où on note :

- Une dynamique moyenne à faible énergie avec une uniformité, régularité et constance dans son l'intensité, en plus d'une forte agitation localement (Plages d'Emsa, Azla et le sud de M’diq), suite à une double action marine marquée par l'action de la dérive littorale générale (S-N) combinée à la diffraction des plans des vagues par les caps dans ces sites,

- Un test de Visher, montrant une répartition quasi uniforme des éléments moyens et un mode de transport prépondérant par saltation, conjugué localement à une suspension et charriage,

- une érosion continue et quasi générale, dans le temps et dans l'espace des sites de Fnideq (Secteur 1, avec un taux moyen global d'érosion de - 78.00 mètres linéaires sur 55 ans, soit un taux moyen annuel d'environ - 1,42 mètres linéaires/an), Cabo Negro, Sidi Abdeslam, Azla, Emsa (Secteur 2, avec un taux d'érosion moyen global de - 82.80 mètres linéaires sur 55 ans, soit un taux d'érosion annuel moyen de l'ordre de - 1.50 mètres linéaires/an),

- Une accrétion limitée des sites de Restinga, Kabyla, M’diq (Secteur 1, avec un taux moyen global d'accrétion est estimé à +87.00 mètres linéaires sur 55 ans, soit un taux moyen annuel d'environ $+1,58$ mètres linéaires/an), et Martil (Secteur 2, avec un taux 


\section{Thème 2 - Dynamique sédimentaire}

d'accrétion moyen global d'environ + 37.50 mètres linéaires sur 55 ans, soit un taux d'accrétion annuel moyen de l'ordre de +0.68 mètres linéaires/an),

- Une dynamique marine, à trois composantes, à savoir : Les courants perpendiculaires (Houles des secteurs $\mathrm{E}$ et $\mathrm{NE}$ ), les marées semi diurnes de faible amplitude et les courants de la dérive littorale, d'intensité moyenne et de direction ( $\mathrm{S}-\mathrm{N}$ : Province septentrionale),

De même, sur le littoral atlantique marocain de la région de Tanger à l'Ouest de la région de Ksar Esghir (Kerroum, 2013), on note l'existence de :

- Une évolution résultantes (entre 1963 et $2001=48$ ans), à tendance positive avec environ 69 \% des espaces en accrétion contre 31 \% d'érosion des espaces littoraux de la région.

- Un taux d'accrétion global du trait de l'ordre de 80 m sur 48 ans avec une vitesse moyenne annuelle d'accrétion d'environ +1 ,67 mètre linéaire/an,

- Un taux d'érosion de la ligne de rivage d'environ 26 m/48 ans avec une vitesse moyenne annuelle de recul de l'ordre de - 0,54 mètres linéaires/an

- Une stabilité touchant pour l'essentiel les zones des falaises.

Au terme de cette étude, il parait évident, que l'évolution historique et morphologique de la ligne de rivage des littoraux de la région entre Tanger - Ksar Esghir - Tétouan, est régi par le même contexte hydrodynamique, climato-hydrologique et géologique.

\section{Références bibliographiques}

BENALI H., EL MOUTCHOU B. (2013). Evolution géomorphologique des reliefs du bassin versant de Ksar Esghir (Province de Tanger - NW du Maroc). $1^{\text {ér }}$ Rencontre des Sciences Géomantiques. Rabat.

CHALOUAN A (1986). Les nappes ghomarides (Rif septentrional Maroc). Un terrain varisque dans la chaîne alpine. Thèse Doc. Sc., Univ. Strasbourg, 317 p.

DIDON J., DURAND-DELGA M., KORNPROBST J. (1973). Homologies géologiques entre les deux rives du détroit de Gibraltar. Bull. Soc. géol. Fr., 7, XV, pp 77-105. http://dx.doi.org/10.2113/gssgfbull.S7-XV.2.77

DURAND-DELGA M., FONTBOTE J.M. (1980). Le cadre structural de la Méditerranée occidentale. 26th Intern. Geol. Congress, Les chaînes alpines issues de la Téthys, Mém BRGM, Vol. 11, pp 67- 85.

DURAND-DELGA M., HOTTINGER L., MARCAIS J., MATTAUER M., MILLIARD Y., SUTER G. (1960-62). Données actuelle sur la structure de rif. In Livre-mémoire P. Fallot, Mém. S.G.F., Paris, t. 1, pp 399-422.

EL FADEL L (2006). Evolution morpho-dynamique et analyse morphostructurale du littoral méditerranéen marocain de Oued Laou (Provinces de Tétouan et Chefchaouen Maroc Nord Occidental). Mémoire de DESA. Univ. Abdelmalek Essaâdi, 76 p 


\section{XIV $V^{\text {èmes }}$ Journées Nationales Génie Côtier - Génie Civil \\ Toulon, 29 juin au $1^{\text {er }}$ juillet 2016}

EL MOUTCHOU B. (1995). Dynamique côtière actuelle et évolution morpho dynamique de la frange littorale entre M'diq et Oued Laou (province de Tétouan, Maroc Nord Occidental). Thèse de 3ème Cycle, Univ. Mohamed V, Rabat, 165 p.

EL MOUTCHOU B. (2014). Caractérisation morphosédimentaire, morphodynamique, géomorphologique et dynamique des versants du littoral méditerranéen marocain entre Fnideq et Kaa Asresse (Provinces de Tétouan et Chefchaouen, Maroc Nord Occidental). Thèse d'Etat, Université Abdelmalek Essaadi-Fac. Sc. Tétouan. 305 p EL MOUTCHOU B. EL FADEL L., EL HAJJAJI KH. (2011b). Evolution morphodynamique et morphosédimentaire du littoral méditerranéen d'Oued Laou (Tétouan - Maroc). Conférence méditerranéenne côtière et maritime. Edition 2. Tanger. Maroc, pp 157-162. http://dx.doi.org/10.5150/cmcm.2011.034

EL MOUTCHOU B., EL MEKADEM N., EL FADEL L., EL HAJJAJI KH. (2011a). Evolution historique et géomorphologique de la ligne du rivage de la zone côtières de Bou Ahmed (Chefchaouen - Maroc). Conférence méditerranéenne côtière et maritime. edition 2. Tanger. Maroc, pp 163-168. http://dx.doi.org/10.5150/cmcm.2011.035

FOLK R.L., WARD W.C. (1957). Brazos river bar: a staday in the significance of grain size parameters. J. Sedim. Petrol., Vol. 27(1), pp 3-26. http://dx.doi.org/10.1306/74D70646-2B21-11D7-8648000102C1865D

JAAIDI E.B., AHMAMOU M., ZOUGARY R., CHATRE B., EL MOUTCHOU B., MALEK F., NAIM K. (1993). Le littoral méditerranéen entre Tétouan et Ceuta et atlantique entre Tanger et Asilah (Maroc) : Impact des aménagements portuaires sur la dynamique côtière. In Aménagement du littoral et évolution des côtes; L'environnement côtier marocain en péril. Publ, Com, Nat, Géogr, Maroc, Sept 1993, pp 21-34.

KERROUM A., (2013) : Etude morphodynamique et occupation du sol de la façade atlantique de la région de Tanger (Province de Tanger - Maroc). Mémoire de Master, Univ. Abdelmalek Essaadi, FS-Tétouan, 75p.

MASSELINK G., HEGGE B. (1995). Morphodynamics of meso- and macrotidal beaches: Examples from central Queensland, Australia. Marine Geology, Vol. 129: pp 1-23. http://dx.doi.org/10.1016/0025-3227(95)00104-2

NORDSTROM K.F. (1977). The use of grain size statistics to distinguish between high and moderate energy beach sediments. Journal Sedimentary Petrology, Vol. 47, pp 1287-1294

PAIDAR.MED (1996). Programme d'Action Intégré pour le Développement et l'Aménagement de la Région Méditerranéenne Marocaine. Diagnostic, Volume IV, DAT.

VISHER G.S. (1969). Grain size distributions and depositional processes. Journal of Sedimentary Petrology, Vol. 39, pp 1074-1106 
Thème 2 - Dynamique sédimentaire 